Quim. Nova, Vol. 26, No. 1, 10-12, 2003

\title{
DETERMINAÇÃO DE $\alpha$-TOCOFEROL EM ALHO IRRADIADO UTILIZANDO CROMATOGRAFIA LÍQUIDA DE ALTA EFICIÊNCIA (CLAE)
}

\author{
Magda Dias Gonçalves Rios* e Marilene De Vuono Camargo Penteado \\ Departamento de Alimentos e Nutrição Experimental, Faculdade de Ciências Farmacêuticas, Universidade de São Paulo,
} Av. Professor Lineu Prestes, 580, Bloco 14, 05508-900 São Paulo - SP

Recebido em 20/8/01; aceito em 1/8/02

\begin{abstract}
DETERMINATION OF $\alpha$-TOCOPHEROL (VITAMIN E) IN IRRADIATED GARLIC BY HIGH PERFORMANCE LIQUID CHROMATOGRAPHY (HPLC). The effects of ${ }^{60} \mathrm{Co}$ ionizing radiations in doses of 0, 75, 100, 150, 200 and 250Gy on garlic, upon the $\alpha$-tocopherol concentration were studied. The $\alpha$-tocopherol contents were established by high performance liquid chromatography (HPLC), after direct hexane extraction from the garlic samples. The $\alpha$-tocopherol was determined through normalphase column, and mobile phase was composed by hexane: iso-propyl alcohol (99:01 v/v), with $2 \mathrm{~mL} / \mathrm{min}$ flow rate and fluorescence detector. It is statistically shown that an irradiation dose of up to $150 \mathrm{~Gy}$ does not affect the garlic $\alpha$-tocopherol content.
\end{abstract}

Keywords: garlic; $\alpha$-tocopherol; HPLC.

\section{INTRODUÇÃO}

O alho, conhecido botanicamente como Allium sativum L., possui características acentuadas de aroma e sabor que lhe atribuem propriedades condimentares que, há muito, conferem a esta hortaliça destaque na culinária mundial e particularmente na brasileira. Além destas formas de utilização, o alho tem sido matéria-prima muito utilizada na indústria farmacêutica, por possuir características de um alimento funcional ${ }^{1,2}$.

As condições climáticas, solos, variedades e técnicas de manuseio e armazenamento pós-colheita influenciam a composição química e, conseqüientemente, a qualidade do alho, alterando sua adequação ao consumo caseiro ou industrial ${ }^{3,4}$.

Durante o armazenamento, ocorrem perdas de peso e alterações na composição química dos bulbos relacionadas à transpiração, ao brotamento e aos ataques de pragas na fase pós-colheita ${ }^{5}$.

Desde que haja um bom gerenciamento na pós-colheita do alho, ele poderá ser estocado por longos períodos, sem grandes perdas na sua composição química. Isto é possível pela aplicação de diferentes métodos que estendam a dormência, na qual o bulbo inicia a fase de brotamento. Durante este último período há um aumento dos processos catabólicos nos tecidos de armazenamento, os compostos nutritivos são transportados para o broto, e a consequiência desta mobilização é a perda da qualidade do alho, devido às perdas das propriedades organolépticas e de hidratação ${ }^{6}$.

O uso da radiação ionizante em alhos tem o propósito de inibir o brotamento e reduzir a perda de peso durante o período da estocagem ${ }^{7}$.

A radiação ionizante é uma técnica bastante utilizada em diversos países para preservação dos alimentos, tanto in natura, como os processados industrialmente.

Muitos países, incluindo os em desenvolvimento, estão utilizando alimentos irradiados para a redução de perdas na pós-colheita, que são as causas fundamentais da escassez de alimentos.

Alimentos irradiados são aqueles que foram tratados com um determinado tipo de radiação ionizante, em condições de segurança controlada, para se obterem algumas propriedades convenientes, tais como: inibição do brotamento; redução de organismos patogênicos;

*e-mail: riosmagda@hotmail.com eliminação de insetos e parasitas, entre outras, com o objetivo de manter a boa qualidade do alimento por mais tempo.

A exposição do alimento à radiação ionizante pode resultar em alterações na composição das vitaminas, sendo que a vitamina $\mathrm{E}(\alpha-$ tocoferol) é bastante sensível a radiação ionizante . $^{8}$

A cromatografia líquida de alta eficiência (CLAE) tem se mostrado uma técnica eficiente na separação e quantificação do $\alpha$ tocoferol, embora o principal problema desta determinação seja a padronização das condições de extração, características físico-químicas e estabilidade da vitamina ${ }^{9}$.

No Brasil, os alimentos irradiados começam agora a ser uma realidade, fazendo-se necessário analisar cuidadosamente todas as alterações que possam vir a ser relacionadas a estes alimentos. Assim, a proposta deste trabalho foi a determinação e quantificação dos teores de $\alpha$-tocoferol (vitamina E) em amostras de alho irradiado, devido à grande importância desta vitamina na manutenção da saúde humana.

\section{MATERIAIS E MÉTODOS}

\section{Amostras}

Para as análises foram utilizados alhos (Allium sativum L.) do grupo roxo, sub-grupo nobre, classe 4, tipo especial, adquiridos em mercados da cidade de São Paulo no período de 1999 a 2000 e mantidos à temperatura ambiente.

\section{Reagentes e outros materiais}

Reagentes: $\alpha$-tocoferol (T-3251-Sigma), metanol (p.a), hidróxido de potássio (p.a), acetonitrila, clorofórmio, hexano e isopropanol (para cromatografia) sendo todos da marca Merck.

A fase móvel foi filtrada com membrana 0,45 $\mu \mathrm{m}$ marca Millipore (FHUP04700). Para filtração das amostras foram utilizadas unidades filtrantes HV Millex, 0,45 $\mu \mathrm{m}$ de porosidade, da Millipore (JBR610222).

As colunas cromatográficas utilizadas foram: sílica, marca

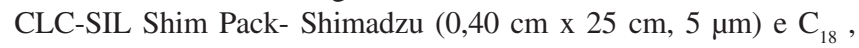
$5 \mu \mathrm{m}, 250 \mathrm{~mm}$ x 4,6 mm, da marca Vydac 201TP54. 


\section{Equipamentos}

Para o preparo das amostras e das fases móveis foram utilizados os seguintes equipamentos:

- Espectrofotômetro marca Beckman, modelo DU-70

- Balança analítica marca A\&D, modelo HA - 202M

- Balança semi-analítica, marca Marte, modelo AS 2000

- Evaporador rotativo, marca Heidolph WB 2000

- Microtriturador marca Tecnal , modelo Turratec TE102

- Degaseificador (gás Hélio), marca Shimadzu, modelo DGU-2A

As análises por CLAE foram efetuadas em cromatógrafo líquido da marca Shimadzu, composto de bomba (LC-10ADVP), injetor automático (SIL-10ADVP), forno com temperatura controlada (CTO10ASVP), detectores de fluorescência (RF-10AXL) e de arranjo de fotodiodo (SPD-M10AVP) e computador com Software (CLASSVP 5.032) para aquisição dos dados.

\section{MÉTODOS}

\section{Irradiação das amostras}

A irradiação das amostras foi realizada pela Empresa EMBRARAD (Empresa Brasileira de Radiações) em fonte de ${ }^{60} \mathrm{Co}$, Nordion JS-700, com atividade aproximadamente de $1500 \mathrm{kCi}$ e uma taxa de dose de $2,7 \mathrm{kGy} / \mathrm{h}$. As doses utilizadas na irradiação das amostras para a análise do $\alpha$-tocoferol foram: $0 ; 75 ; 100,150 ; 200$ e $250 \mathrm{~Gy}^{10}$. As amostras foram processadas em embalagens de sacos plásticos de $250 \mathrm{~g}$ em duplicata, para cada dose proposta, e mantidas à temperatura ambiente.

\section{Determinação do $\alpha$-tocoferol}

\section{Saponificação}

O método de saponificação foi baseado no procedimento de ALAbdulaly e Simpson ${ }^{11}$. Antes da saponificação os alhos foram descascados com faca e triturados em liqüidificador, ambos de modelo doméstico. Foram pesados cerca de $5 \mathrm{~g}$ da amostra. Às amostras, foram adicionados $30 \mathrm{~mL}$ de $\mathrm{KOH}$ metanólico $15 \%$ (m/v). A mistura permaneceu uma noite no escuro sob atmosfera de nitrogênio.

A mistura foi transferida para um funil de separação sendo adicionados cerca de $50 \mathrm{~mL}$ de éter etílico, ficando em repouso por 5 min para completa separação entre as fases. A fase inferior (metanólica) foi recolhida e a etapa de extração repetida duas vezes, com tempo de agitação de $10 \mathrm{~min}$ cada uma. Os extratos etéreos foram combinados e lavados com água destilada até a remoção da água residual. A seguir, o éter etílico foi removido em evaporador rotativo. Ao resíduo foram adicionados $100 \mathrm{~mL}$ de hexano: isopropanol 99:01 v/v. Esta solução foi filtrada em membrana de $0,45 \mu \mathrm{m}$, sendo injetados $10 \mu \mathrm{L}$ no cromatógrafo.

\section{Extração}

Os métodos de extração foram baseados nos procedimentos de Gong e Ho ${ }^{12}$, Malik et al. ${ }^{2}$, Al-Abdulaly e Simpson ${ }^{11}$, com modificações.

As amostras foram trituradas previamente em um liqüidificador e, em seguida, foram pesados cerca de $5 \mathrm{~g}$ da amostra, e adicionados $20 \mathrm{~mL}$ do solvente extrator, sendo testados hexano, heptano e éter etílico. As amostras foram novamente trituradas usando microtriturador com tempo de 2 min cada uma, e a etapa de extração repetida 3 vezes. A seguir, o solvente foi removido em evaporador rotativo. Ao resíduo foram adicionados $100 \mathrm{~mL}$ de hexano: isopropanol 99:01 v/v (fase móvel). Esta solução foi filtrada em membrana de $0,45 \mu \mathrm{m}$, sendo injetados $10 \mu \mathrm{L}$ no cromatógrafo.

\section{Condições cromatográficas}

Foram testados métodos cromatográficos de fase reversa e normal.

O método de fase reversa, segundo Malik et al. ${ }^{2}$, utilizou sistema isocrático, coluna $\mathrm{C}_{18}, 5 \mu \mathrm{m}, 250 \mathrm{~mm}$ x 4,6 mm, da marca Bakerbond. Para testar este método, utilizou-se uma coluna com as mesmas especificações, porém de marca diferente (Vydac 201TP54). A fase móvel foi composta de acetonitrila: metanol: clorofórmio (47:47:6 v/v/v), vazão de 1,5 mL/min. Foi utilizado o detector UV a $292 \mathrm{~nm}$ para determinação da vitamina $\mathrm{E}$.

$\mathrm{O}$ método de fase normal com eluição isocrática baseou-se na literatura ${ }^{13-15}$ com fase móvel hexano: isopropanol (99:01v/v), vazão de $2 \mathrm{~mL} / \mathrm{min}$ e detecção por fluorescência (lambda excitação = $295 \mathrm{~nm}$ e lambda emissão $=330 \mathrm{~nm})^{15}$.

As fases móveis foram filtradas e degaseificadas durante $10 \mathrm{~min}$.

\section{RESULTADOS E DISCUSSÃO}

\section{Avaliação das condições utilizadas na determinação do $\alpha$-tocoferol}

Apesar de ser um procedimento bastante utilizado, pode-se verificar na Tabela 1 que a saponificação seguida de extração com éter etílico é o método de menor eficiência para recuperação da fração lipossolúvel. Para esta determinação, foi utilizada a cromatografia líquida de fase normal, conforme método ${ }^{13-15}$ descrito anteriormente.

Na Figura 1c está apresentado o perfil cromatográfico do padrão de $\alpha$-tocoferol em coluna de fase normal .

Este método proporcionou boa detecção para o $\alpha$-tocoferol da amostra de alho, conforme Figura 1b. A utilização da cromatografia de fase reversa ${ }^{2}$ foi descartada, pois não propiciou boa resolução para o $\alpha$-tocoferol da amostra (Figura 1a) .

Os resultados das análises por CLAE mostraram que o alho contém teores significativos de $\alpha$-tocoferol, próximos aos encontrados na literatura ${ }^{2,16}$.

A análise estatística mostrou diferenças $(\mathrm{P}>0,05)$ entre os resultados da Tabela 1 para as extrações com hexano, heptano e éter etílico.

Tabela 1. Comparação dos teores do $\alpha$-tocoferol determinados por CLAE em amostras de alho utilizando saponificação e diferentes solventes de extração

\begin{tabular}{lcc}
\hline Variáveis (*) & $\begin{array}{c}\text { Médias }(* *) \\
\mu \mathrm{g} \text { de } \alpha \text {-tocoferol por } \\
\mathrm{g} \text { de amostra }\end{array}$ & $\begin{array}{c}\text { Estimativa do } \\
\text { Desvio padrão }\end{array}$ \\
\hline Éter Etílico (CS) & 2,65 & 0,29 \\
Éter Etílico (SS) & 5,66 & 0,22 \\
Heptano (SS) & 5,92 & 0,13 \\
Hexano (SS) & 6,95 & 0,08 \\
\hline
\end{tabular}

(*) $\mathrm{CS}=$ saponificação seguida de extração; $\mathrm{SS}=$ extração direta com solvente; (**) média de 3 amostras em duplicata

A curva analítica do $\alpha$-tocoferol apresentou boa linearidade dentro das faixas de concentrações estudadas (50 a 150\% do nível nominal de concentração da amostra). A regressão linear para esta curva, $\mathrm{y}=$ $\mathrm{A}+\mathrm{Bx}$, onde $\mathrm{A}=$ coeficiente linear, $\mathrm{B}=$ coeficiente angular, $\mathrm{r}^{2}=$ coeficiente de correlação, foi: $y=6.10^{-8} \cdot x+0,0008, r^{2}=0,9954$.

Verificou-se que a adição de padrão diluído em solvente à amostra antes do procedimento de análise, propiciou a recuperação de 95,30\% do $\alpha$-tocoferol estudadas (as amostras foram preparadas em três níveis de concentração na faixa de $50 \%$ a $150 \%$ da quantidade nominal da amostra) ${ }^{17}$. 

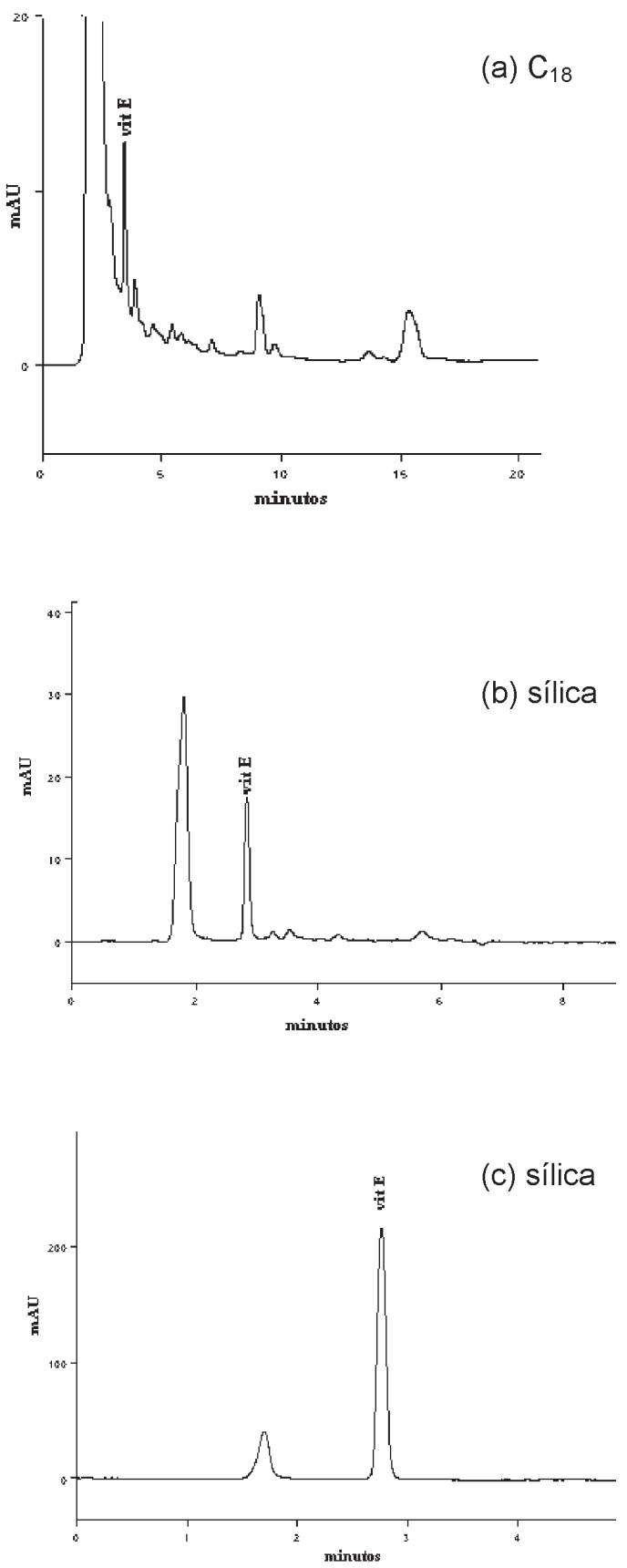

Figura 1. Cromatogramas do $\alpha$-tocoferol em alhos (a) fase móvel: acetonitrila: metanol: clorofórmio (47: 47: $6 \mathrm{v} / \mathrm{v} / \mathrm{v}$ ); coluna $C_{18}$; vazão: 1,5 $\mathrm{mL} / \mathrm{min}$; detector $U V(292 \mathrm{~nm}) ; T_{R}=3,44 \mathrm{~min}$. (b) fase móvel: hexano: isopropanol (99:01 v/v); coluna de sílica; vazão: $2 \mathrm{~mL} / \mathrm{min}$; detector de fluorescência $(\lambda e x=295 \mathrm{~nm}$ e $\lambda$ em $=330 \mathrm{~nm}) ; T_{R}=2,80 \mathrm{~min}$. (c) Padrão de $\alpha$-tocoferol, condições iguais a (b)

Os valores obtidos para os limites de detecção (LD) e quantificação (LQ) do $\alpha$-tocoferol foram: $\mathrm{LD}=0,59 \mu \mathrm{g} / \mathrm{mL}$ e $\mathrm{LQ}=$ $0,98 \mu \mathrm{g} / \mathrm{mL}$. LD e LQ foram obtidos das áreas de picos do padrão com, respectivamente, três e cinco vezes a altura do ruído ${ }^{18,19}$.

Os valores de $\alpha$-tocoferol com e sem aplicação da radiação gama, estão apresentados na Tabela 2.

Os teores de $\alpha$-tocoferol obtidos variaram entre 6,41 - 6,87 (Tabela 2), estes resultados foram similares àqueles relatados por Malik et al. ${ }^{2}$ que trabalharam na análise de $\alpha$-tocoferol em alhos por CLAE.
Tabela 2. Efeitos da irradiação sobre os teores do $\alpha$-tocoferol em alho determinados por CLAE

\begin{tabular}{lcc}
\hline $\begin{array}{l}\text { Dose Gy de } \\
\text { radiação ionizante }\end{array}$ & $\begin{array}{c}\alpha \text {-tocoferol }{ }^{(*)} \\
\mu \mathrm{g} / \mathrm{g}\end{array}$ & $\begin{array}{c}\text { Estimativa do } \\
\text { Desvio padrão }\end{array}$ \\
\hline $0^{\mathrm{a}}$ & 6,87 & 0,18 \\
$75^{\mathrm{a}}$ & 6,86 & 0,24 \\
$100^{\mathrm{a}}$ & 6,84 & 0,10 \\
$150^{\mathrm{a}}$ & 6,83 & 0,14 \\
$200^{\mathrm{b}}$ & 6,50 & 0,16 \\
$250^{\mathrm{b}}$ & 6,41 & 0,11 \\
\hline
\end{tabular}

(*) média de 4 amostras com duplicata; ${ }^{a}$ não houve diferença significativa $(\mathrm{P}<0,05) ;{ }^{\mathrm{b}}$ houve diferença significativa $(\mathrm{P}<0,05)$

De acordo com Kwon et al. ${ }^{10}$ a dose de radiação ionizante mínima necessária para evitar o brotamento no alho é de 75Gy, sendo assim, verificamos que não houve perdas nos teores de $\alpha$-tocoferol, quando o alimento é submetido a esta dose.

\section{CONCLUSÕES}

A extração direta foi mais satisfatória que o procedimento mais complexo de saponificação seguido de extração, mostrando-se uma metodologia rápida e eficiente para a recuperação do $\alpha$-tocoferol.

$\mathrm{O}$ hexano foi o extrator mais eficiente para a recuperação do $\alpha$ tocoferol nas amostras analisadas.

A cromatografia de fase normal com sistema de eluição isocrática foi adequada para a resolução do $\alpha$-tocoferol.

$\mathrm{O}$ efeito da radiação ionizante sobre os teores de $\alpha$-tocoferol em alho mostrou diferenças $(\mathrm{P}<0,05)$ somente quando as doses aplicadas foram de 200 e $250 \mathrm{~Gy}$.

\section{AGRADECIMENTOS}

À EMBRARAD (Empresa Brasileira de Radiações) pelo tratamento das amostras. Ao professor D. M. Vizeu pelo apoio técnico.

\section{REFERÊNCIAS}

1. Sato, T. M. D.; Miyata, G. M. D.; Nutrition 2000, 16, 787.

2. Malik, M. N.; Fenko, M. D.; Shiekh, A. M.; Wisniewski, H. M.; J. Agric. Food Chem. 1997, 45, 817.

3. Carvalho, V. D.; Chalfoun, S. M.; Steiner, E. G.; Leite, I. P.; Pesqui. Agropecu. Bras. 1987, 22, 733.

4. Foda, S. A.; Agric. Res. Rev. 1977, 85, 177.

5. Carvalho, V. D.; Souza, S. M. C.; Abreu, C. M. P.; Chagas, S. J. R.; Pesqui. Agropecu. Bras. 1991, 26, 1679.

6. Pellegrini, C. N.; Croci, C. A.; Orioli, G. A.; Radiat. Phys. Chem. 2000, 57,315 .

7. Curzio, O. A.; Croci, C. A.; Radiat. Phys. Chem. 1988, 31, 203.

8. Kilcast, D; Food Chem. 1994, 49, 157.

9. Rizzolo, A.; Polesello, S.; J. Chromatogr. 1992, 624, 103.

10. Kwon, J. H.; Byun, M. W.; Cho, H. O.; J. Food Sci. 1985, 50, 379.

11. AL-Abdulaly, A. B.; Simpson, K. L.; J. Micronutr. Anal. 1989, 5, 161.

12. Gong, B. Y.; Ho, J. W. ; J. Liq. Chromatogr. Relat. Technol. 1997, $20,2389$.

13. Presoto, A. E. F.; Rios, M. D. G.; Almeida-Muradian, L. B.; Boll. Chim. Ig., Parte Sci. 2000, 51, 111.

14. Rios, M. D. G.; Dissertação de Mestrado, Universidade de São Paulo, Brasil, 2001.

15. Bianchini, R.; Penteado, M. V. C.; Cienc. Tecnol. Aliment. 1997, 19, 349.

16. Souci, S. W., Fachmann, W.; Kraut, H.; Food Composition and Nutrition Tables, London: Medpharm, 1994, p. 678.

17. Dadgar, D.; Kelly, M.; Analyst 1988, 113, 229.

18. Moreno, P.; Salvadó, V.; J. Chromatogr., A 2000, 870, 207.

19. Snyder, L. R.; Kirkland, L. L.; Introduction to Modern Liquid Chromatography, $2^{\text {nd }}$ ed., Wiley: New York, 1979, cap. 5. 